\title{
An instrument for studying granular media in low-gravity environment
}

Cite as: Rev. Sci. Instrum. 89, 075103 (2018); https://doi.org/10.1063/1.5034061

Submitted: 09 April 2018 . Accepted: 12 June 2018 . Published Online: 05 July 2018

S. Aumaître, R. P. Behringer, A. Cazaubiel, E. Clément, J. Crassous, D. J. Durian (D, E. Falcon (iD, S. Fauve, D. Fischer, A. Garcimartín (D), Y. Garrabos, M. Hou, X. Jia, C. Lecoutre, S. Luding, D. Maza, M. Noirhomme, E. Opsomer, F. Palencia, T. Pöschel (D), J. Schockmel, M. Sperl (D), R. Stannarius (D, N. Vandewalle, and P. Yu

\section{ARTICLES YOU MAY BE INTERESTED IN}

Improving underwater localization accuracy with machine learning

Review of Scientific Instruments 89, 074902 (2018); https://doi.org/10.1063/1.5012687

A high-precision and fast-sampling frequency measurement method based on FPGA carry chain for airborne optically pumped cesium magnetometer

Review of Scientific Instruments 89, 075001 (2018); https://doi.org/10.1063/1.5036822

DESIREE electrospray ion source test bench and setup for collision induced dissociation experiments

Review of Scientific Instruments 89, 075102 (2018); https://doi.org/10.1063/1.5030528 


\title{
An instrument for studying granular media in low-gravity environment
}

\author{
S. Aumaître,${ }^{1,2}$ R. P. Behringer, ${ }^{3}$ A. Cazaubiel, ${ }^{4}$ E. Clément,${ }^{5}$ J. Crassous, ${ }^{6}$ D. J. Durian, ${ }^{7}$ \\ E. Falcon,,${ }^{4, a)}$ S. Fauve, ${ }^{8}$ D. Fischer, ${ }^{9}$ A. Garcimartín, ${ }^{10}$ Y. Garrabos,${ }^{11}$ M. Hou, ${ }^{12}$ X. Jia,${ }^{13}$ \\ C. Lecoutre, ${ }^{11}$ S. Luding, ${ }^{14}$ D. Maza,${ }^{10}$ M. Noirhomme, ${ }^{15}$ E. Opsomer, ${ }^{15, b)}$ F. Palencia, ${ }^{11}$ \\ T. Pöschel, ${ }^{16}$ J. Schockmel, ${ }^{15}$ M. Sperl, ${ }^{17}$ R. Stannarius,${ }^{9}$ N. Vandewalle, ${ }^{15, c)}$ and P. Yu ${ }^{14}$ \\ ${ }^{1}$ SPEC, DSM, CEA-Saclay, CNRS URA 2464, F-91191 Gif-sur-Yvette, France \\ ${ }^{2}$ Laboratoire de Physique, ENS Lyon, UMR-CNRS 5672, F-69007 Lyon, France \\ ${ }^{3}$ Department of Physics, Duke University, Durham, North Carolina 27708-0305, USA \\ ${ }^{4}$ Université Paris Diderot, SPC, MSC, UMR 7057 CNRS, F-75013 Paris, France \\ ${ }^{5}$ PMMH, ESPCI, UMR 7636 CNRS, F-75005 Paris, France \\ ${ }^{6}$ Université Rennes 1, IPR, UMR 6251 CNRS, F-35042 Rennes, France \\ ${ }^{7}$ University of Pennsylvania, Philadelphia, Pennsylvania 19104-6396, USA \\ ${ }^{8}$ École Normale Supérieure, LPS, CNRS, UMR 8550, F-75005 Paris, France \\ ${ }^{9}$ IEP, Otto von Guericke Universität, D-39106 Magdeburg, Germany \\ ${ }^{10}$ DFMA, Universidad de Navarra, E-31080 Pamplona, Spain \\ ${ }^{11}$ CNRS, ICMCB, Université de Bordeaux, UMR 5026, F-33600 Pessac, France \\ ${ }^{12}$ Institute of Physics, Chinese Academy of Sciences, Beijing 100190, China \\ ${ }^{13}$ Institut Langevin, ESPCI Paris, PSL, CNRS, F-75005 Paris, France \\ ${ }^{14}$ MSM, University of Twente, 7500 AE Enschede, The Netherlands \\ ${ }^{15}$ GRASP, Institute of Physics B5a, University of Liège, B-4000 Liège, Belgium \\ ${ }^{16}$ Friedrich-Alexander Universität, IMS, D-91052 Erlangen, Germany \\ ${ }^{17}$ Institut für Materialphysik im Weltraum, DLR, D-51170 Köln, Germany
}

(Received 9 April 2018; accepted 12 June 2018; published online 5 July 2018)

\begin{abstract}
A new experimental facility has been designed and constructed to study driven granular media in a low-gravity environment. This versatile instrument, fully automatized, with a modular design based on several interchangeable experimental cells, allows us to investigate research topics ranging from dilute to dense regimes of granular media such as granular gas, segregation, convection, sound propagation, jamming, and rheology — all without the disturbance by gravitational stresses active on Earth. Here, we present the main parameters, protocols, and performance characteristics of the instrument. The current scientific objectives are then briefly described and, as a proof of concept, some first selected results obtained in low gravity during parabolic flight campaigns are presented. Published by AIP Publishing. https://doi.org/10.1063/1.5034061
\end{abstract}

\section{INTRODUCTION}

Granular systems belong to a particular class of materials since grains may exhibit solid, fluid, or gaseous behaviors. Although nearly $80 \%$ of the products used in industry are powders and grains, many fundamental questions concerning their rheology and their dynamics are still unsolved. ${ }^{1}$ Space exploration (such as regolith on asteroid surfaces, powder propellants of rockets, or planetary rings dynamics) and space exploitation (such as asteroid mining) will also face major challenges concerning the handling of granular materials in low-gravity environments. ${ }^{2-4}$ For example, nobody knows how to perform a simple operation like sieving in space, whereas several month efforts failed to free NASA's Mars Exploration Rover Spirit from a Martian sand trap in 2010. Therefore, it is of primary interest to better understand the flow and the dynamics of granular media in low-gravity conditions.

On Earth, when granular matter is subjected to vibrations, liquid-like behavior occurs such as convective flow $^{5}$ or surface

\footnotetext{
a)Electronic mail: eric.falcon@univ-paris-diderot.fr

b)Electronic mail: eric.opsomer@uliege.be

c)Electronic mail: nvandewalle@uliege.be
}

waves. ${ }^{6}$ For strong enough forcing, gas-like behavior, with particular modes of momentum and energy transport, ${ }^{7}$ arises where particles move erratically in response to the vibrations and the dissipative nature of collisions. When the density of particles increases within such a granular gas, the formation of a dense cluster of particles is observed due to inelastic collisions. ${ }^{8-10}$ This cluster formation constitutes one of the most astonishing properties of granular matter under vibrations. When the forcing is stopped, numerical simulations have shown the formation of density gradients during the cooling (instability of the homogeneous cooling state) ${ }^{11}$ as well as inelastic collapse (particles undergoing an infinite number of collisions in finite time). ${ }^{12}$ However, several phenomena occurring on Earth (such as sedimentation, convection, and confinement pressure due to the weight of grains) disappear in low gravity, thus leading to strongly different behavior for granular matter.

Generally, ground based experiments are perturbed by anisotropy induced by gravity and by the friction force which acts on all the particles and which is far from being negligible. A low-gravity environment is, thus, needed for dilute regimes of vibrated granular medium because it is the only way to achieve an experimental situation in which inelastic collisions are the only interaction mechanism. First, 
experiments investigating the dynamics of these granular gases were conducted in the late nineties on a MiniTexus sounding rocket. ${ }^{9}$ More recently, gases of rod-shaped particles have been studied on a REXUS flight ${ }^{13}$ and in the ZARM Drop Tower in Bremen. ${ }^{14}$ For dense regimes, a low-gravity environment is also needed to better understand the rheology, ${ }^{15}$ sound propagation, ${ }^{16}$ and jamming transition ${ }^{17}$ of dense granular media without confinement pressure of grains due to their own weight.

In order to address fundamental questions and potential applications regarding grains in a low-gravity environment, in 2011, the European Space Agency (ESA) created a Topical Team ${ }^{18}$ on the subject. Its objective is to perform unique measurements on granular gas, convection, segregation, sound propagation, and jamming in granular materials subjected to vibrations without the symmetry breaking influence of gravity. In collaboration with DTM Technologies $^{\text {TM }}$ (Modena, Italy), the Topical Team designed the VIP-Gran-PF instrument (short for Vibration-Induced Phenomena in Granular Matter in Parabolic Flights) whose modular design allows us to investigate both dilute and dense granular regimes.

This paper is organized as follows. Section II describes the experimental instrument and its performance. Section III presents the current scientific objectives and, as a proof of concept, some selected results obtained with this facility during four ESA parabolic flight campaigns (PFCs) onboard a modified Airbus A310 Zero-G aircraft. Finally, we draw our conclusions and perspectives in Sec. IV.

\section{INSTRUMENT DESCRIPTION AND PERFORMANCE}

The setup of the VIP-Gran instrument is displayed in Fig. 1. The basic principle of the experiment consists of a closed cell containing particles, in which two opposite walls of the cell are pistons vibrating either in-phase or out-ofphase motions. Control parameters are the number of particles, the cell length (volume), the amplitude, and the frequency of vibrations. Different interchangeable cells can be used depending on the scientific objective. Accelerometers are screwed in the shaft of each vibrating piston. Impact sensors are also implemented to measure the collision statistics of particles impacting on the pistons. Two cameras allow quantitative measurements including particle tracking in dilute regimes and correlations between particle displacements.

We describe below in detail the five major components of the setup: (A) interchangeable experimental cells to be filled with granular material; (B) a bead feeder that allows adding particles into the cells during the experiment; $(\mathrm{C})$ the driving mechanism that allows the energy injection, the control of the volume, and the confining pressure; (D) the accelerometers and impact sensors; and (E) two high resolution speed cameras to capture relevant data during the experiments and the illuminations.

\section{A. Experimental cells}

An experimental cell is a closed cuboidal container of $60 \times 30 \times h \mathrm{~mm}^{3}$ that can be filled by granular material
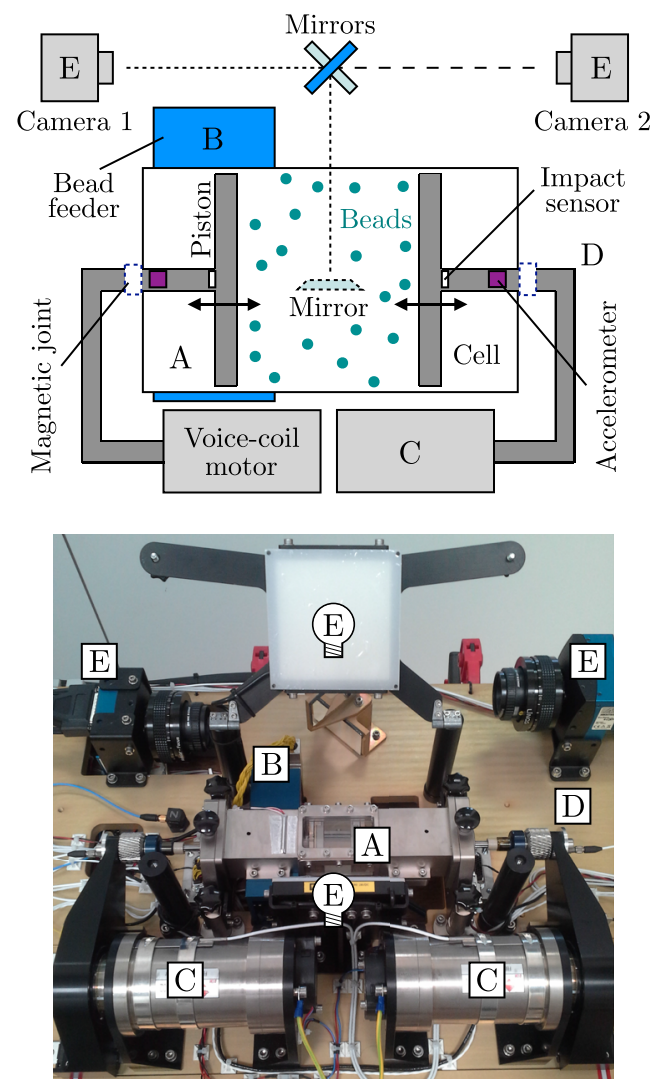

FIG. 1. For letters A, B, C, D, and E, refer to the main text. Top: Sketch (top view) of the VIP-Gran instrument. Two opposite walls, driven by voice coil motors, inject mechanical energy into the experimental cell containing a granular medium. Two cameras track grains or visualize the system from the bottom and from the side. Accelerometers and impact sensors are mounted on the shafts of the pistons respectively to control the energy injection in the system and to measure the collision statistics of the granular medium with the pistons. Bottom: Picture of the instrument built by DTM Technologies for parabolic flight experiments.

(spherical and/or aspherical particles). The top, bottom, and lateral sides of the cells are made of transparent polycarbonate, $8 \mathrm{~mm}$ in thickness. The two opposite walls of the cell are connected magnetically to linear voice-coil motors and act as pistons. The average distance, $L$, between the pistons can be adjusted in order to control the accessible volume of the system. Initially, a three-dimensional (3D) container $(h=30 \mathrm{~mm})$ and a quasi two-dimensional (2D) container $(h=5 \mathrm{~mm})$ were developed. A sketch describing these cells is given in Figs. 2(a) and 2(b). Different modifications can be applied to the cells in order to conduct particular experiments. For instance, placing additional walls within the container, as in Figs. 2(c) and 2(d), to create compartments can lead to trapping. Changing the curvature, or the roughness, of the pistons as in Figs. 2(e) and 2(f) can modify the energy injection into the system. Different physical phenomena and properties can then be investigated in low gravity within specific interchangeable cells: the outof equilibrium stationary states of diluted granular medium in cells (a) and (b), handling of grains in cells (c) and (d), rheology in a cell (e) or sound propagation in granular media under weak applied confinement in a modified cell (a), and behaviors of the rheology of wet grains in a sealed cell (f). Some cells, such as (e) and (f), are in development. Therefore, we limit 
a)

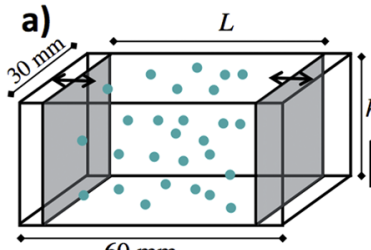

b)

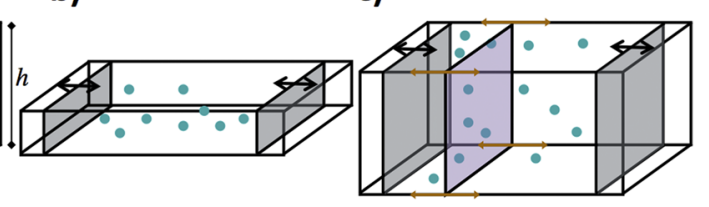

d)

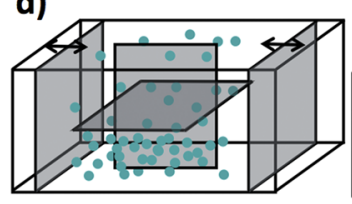

e)

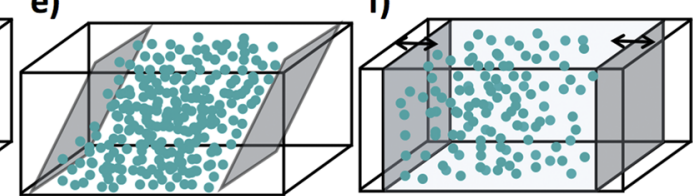

FIG. 2. Sketches of the experimental cells used in VIPGran-PF. Parameter $L$ is tunable, thanks to the two mobile gray walls that act as pistons. The height $h$ is $30 \mathrm{~mm}$ for $3 \mathrm{D}$ cells and $5 \mathrm{~mm}$ in the case of the quasi 2D cells: (a) 3D cell, (b) quasi-2D cell, (c) 3D cell with a free moving wall, (d) 3D partitioned cell, (e) 3D shear cell, and (f) $3 \mathrm{D}$ wet cell. Cells can easily be exchanged, thanks to a magnetic joint connection with the kinematic chain (see Fig. 1). the discussion herein below to the use of the 3D cell (a) and the quasi $2 \mathrm{D}$ one (b).

\section{B. Bead feeder}

The experimental cells described in Sec. II A can be filled by spherical particles of different materials (bronze, steel, aluminum, glass, polyamide, etc.) and different diameters; see Table I. Moreover, cylindrical steel rods were also tested. Granular matter can either be placed directly into the cells before the experiment or be injected into them during the experimental runs using the bead feeder (see Fig. 3). The barrel of the bead feeder is divided into eight chambers, each able to contain either 1000 small $(1 \mathrm{~mm})$ or 250 large $(2 \mathrm{~mm})$ spherical particles. Thanks to a motorized linear rail slide system, each chamber can be positioned in front of the entry hole of the experimental cell. A piston then injects the particles. Note that the bead feeder does not allow extracting grains which means that, to start over again with new initial filling conditions, the cell has to be exchanged.

\section{Driving mechanism}

Two opposite walls of the cell are vibrating pistons (see Fig. 1). They are moved by using two linear voice-coil motors driven by a selected vibration stimulus (sine, single-pulse, quasi-static ramp, etc.). The forcing amplitude, $A$, of the piston is in the range $[0.2,25] \mathrm{mm}$ (peak-to-peak), and its forcing frequency, $f$, in the range $[1,50] \mathrm{Hz}$. Note that the piston acceleration is limited to $5 g$ ( $g$ being the acceleration of Earth

TABLE I. Table of the experimental parameters of the VIP-Gran instrument. In each row, the description and the typical range of values are given for the different parameters. Note that $L$ and $h$ are defined in Fig. 2(a).

\begin{tabular}{llc}
\hline \hline Symbol & \multicolumn{1}{c}{ Description } & Value \\
\hline$N$ & Number of particles & $\ldots$ \\
$R$ & Radius of the particles & $1-5 \mathrm{~mm}$ \\
$L$ & Distance between pistons & $2-60 \mathrm{~mm}$ \\
$h$ & Height of the cell & 5 or $30 \mathrm{~mm}$ \\
$V$ & Volume of the system & $0.3-54 \mathrm{~cm}^{3}$ \\
$\phi$ & Packing fraction & $0 \%-60 \%$ \\
$A$ & Amplitudes of pistons & $0-5 \mathrm{~mm}$ \\
$f$ & Frequencies of pistons & $0-50 \mathrm{~Hz}$ \\
$\varphi$ & Phase shifts of pistons & $0-\pi$ \\
\hline \hline
\end{tabular}

gravity), meaning that some $A, f$ combinations are not possible. For sine vibrations, pistons can oscillate either in-phase or out-of-phase. Both pistons are controlled in displacement with an accuracy of $10 \mu \mathrm{m}$ at an acquisition rate of $1000 \mathrm{~Hz}$. Since the vibrating walls of each cell are connected to the linear motors via magnetic joints mounted on both shafts (see Fig. 1), the cells can be changed on the instrument. A cooling fan is positioned on the back of each motor to keep the temperature constant.

\section{Sensors and acquisition}

Several sensors are installed in the instrument: accelerometers, impact sensors, linear positioning sensors, and temperature sensors. The two mono-axial accelerometers are screwed into the shaft of each vibrating piston to measure the piston's accelerations ( $8 \mathrm{kHz}$ sampling frequency). One triaxial accelerometer is fixed on the breadboard, far away from the cell, in order to measure the ambient acceleration in response to the aircraft motions (1 $\mathrm{kHz}$ sampling rate). For $3 \mathrm{D}$ cells, the impact sensors are rectangular-shaped piezoelectric films mounted behind each vibrating piston, their axis being collinear with the vibration axis. Thanks to a $2 \mathrm{MHz}$ sampling rate, each sensor is able to resolve the collision of a bead impacting on the piston wall. Such a contact lasts a few microseconds since the typical velocity of beads is of the order of few $\mathrm{m} / \mathrm{s}$ at most. The two linear sensors for controlling the piston displacements are acquired at a $3 \mathrm{MHz}$ sampling rate. Four temperature sensors are used to detect, at

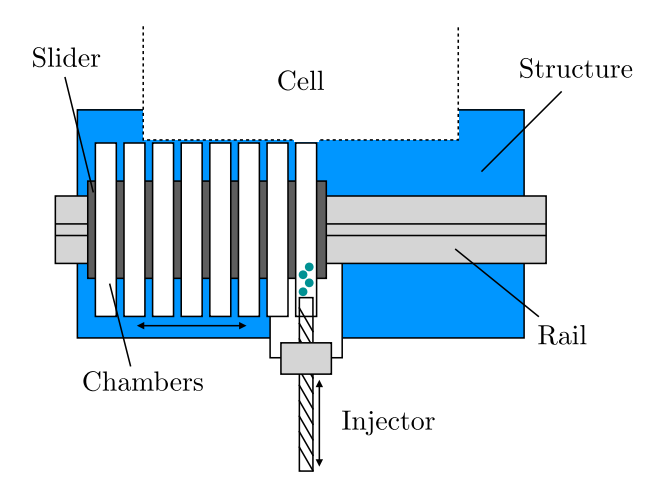

FIG. 3. Sketch of the bead feeder located below the cell. This device is used for the controlled injection of additional granular material into an experimental cell. It corresponds to letter B in Fig. 1. 
a $1 \mathrm{~Hz}$ sampling rate, any abnormal overheating of the experiment cell, of each linear motors, and of the ambient air, to be able to promptly switch off the motors in order to cool them down. The onboard workstation, data acquisition system (interfaced by National Instruments cards), and electronics thus enable, for each parabola, high-frequency acquisitions of both video images and sensor signals as well as programming the required experiment profiles (piston frequency and amplitude and oscillation type). The time-tagging of acceleration data and impact force sensor data is synchronized with image acquisition and piston displacement (with a precision of $10^{-6} \mathrm{~s}$ ).

\section{E. Video cameras and illuminations}

The instrument enables the observation of the experiment by means of two high-speed cameras of frame $1000 \mathrm{fps}$ with a resolution of $1024 \times 1024$ pixels (Mikrotron EoSens 4CXP). The camera frame rate can be chosen down to $1 \mathrm{fps}$ if low-speed acquisition is needed notably for experiments regarding dense regime. Observations are made in two perpendicular directions, perpendicular to the vibration axis. This corresponds to a bottom observation and a side-view observation in Fig. 1. The depth of field is selectable, whereas the field of view covers the full cell during vibrations. At full rates and resolution, 1.4 TBytes of video data are recorded by using both cameras during 31 parabolas ( $22 \mathrm{~s}$ each) of each flight. For each observation direction, the cell is illuminated from the back, corresponding to the two sides perpendicular to the field of view (see Figs. 1 and 4). Note that illuminator 2 on the top of Fig. 1 should be located on the top of the cell but has been turned over to enable the picture. In this open position, this illuminator masks the mirrors to obtain the required optical path between the cameras and the cell (see the top view of Fig. 1). Each illumination is individually switchable, and its intensity adjustable. The light from the light-emitting diode (LED) is diffused and uniform along all field of view. Straight light coming from outside the

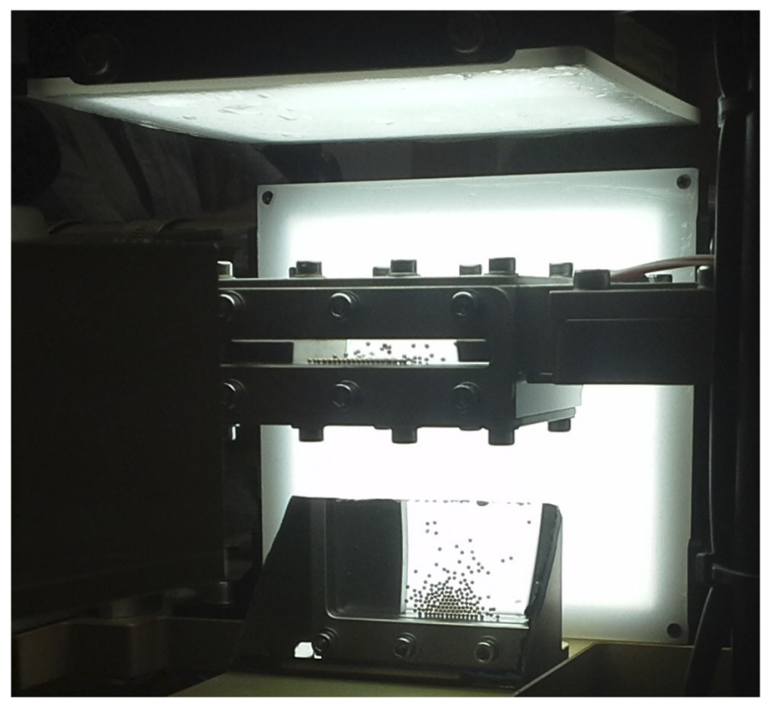

FIG. 4. Snapshot of the quasi-two-dimensional cell in front of the diffuse LED illumination (this image was taken on Earth). On the bottom, one can see the mirror enabling to take pictures from the bottom angle. instrument, reflections on cell glass walls, and bright spots on particles are minimized. We can select which illumination and which camera should be activated (one of them or both).

\section{F. Experimental rack in the aircraft}

A photo of the experimental rack mounted in the aircraft is displayed in Fig. 5. The rack is made of the Bosch Rexroth aluminum profiles, and the instrument baseplate is made of aluminum. The total mass of the experimental rack is $176 \mathrm{~kg}$ (100 kg for the payload, $76 \mathrm{~kg}$ for the structure). The rack dimensions are $1.1 \mathrm{~m} \times 1 \mathrm{~m}$ and $0.67 \mathrm{~m}$ in height. Reinforcement bars of the rack in the aircraft main direction and in the perpendicular direction would resist in the case of a hard landing of $9 g$ and $3 g$, respectively.

\section{G. Low-gravity conditions}

The VIP-Gran-PF instrument was used during five ESA Parabolic Flight Campaigns (PFC63 to PFC67) from November 2015 to November 2017. The first PFC has been dedicated to the testing of the instrument and the other ones to scientific issues. A low-gravity environment (about $\pm 5 \times 10^{-2} \mathrm{~g}$ ) is repetitively achieved by flying with the specially modified
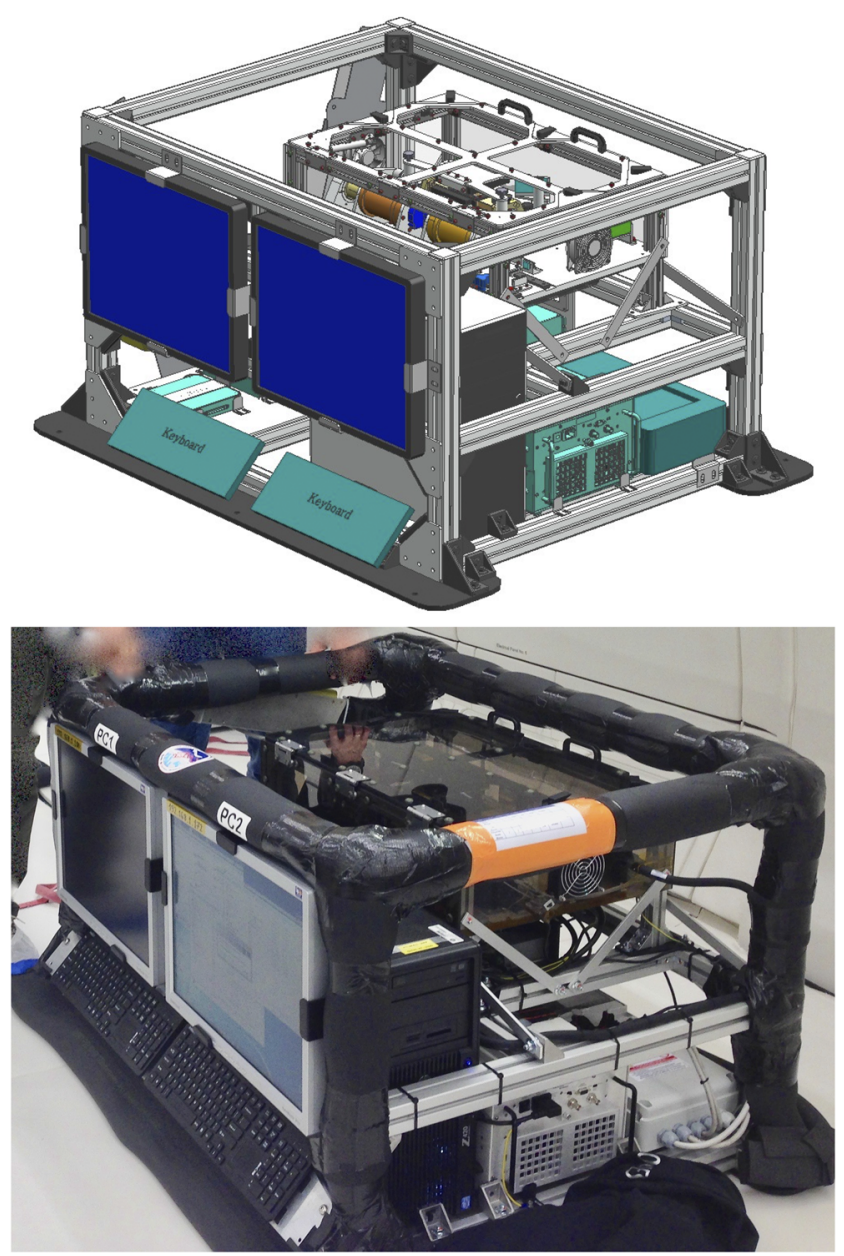

FIG. 5. Top: Drawing of the instrument rack by DTM Technologies. Bottom: Picture of the rack onboard the aircraft. 
Airbus A310 Zero-G aircraft of Novespace ${ }^{19}$ through a series of parabolic trajectories which result in low-gravity periods, each of $22 \mathrm{~s}$. A flight campaign lasts 3 days with 31 low-gravity parabolas per day.

\section{H. Experimental parameters}

Different experiments are performed with various forcing parameters and filling (the number of beads and/or the type of bead). Since the grains cannot be removed automatically from the cell, the different experiments should be classified according to increasing filling parameters. An overview of all relevant experimental parameters for the performed experiments can be found in Table I.

\section{SCIENTIFIC APPLICATIONS}

The VIP-Gran-PF instrument is used since mid-2015. A wide variety of experimental situations can be studied in low-gravity conditions from dilute granular systems to dense assemblies. Several fundamental questions are notably addressed as follows:

(1) How a granular gas (which is intrinsically dissipative due to inelastic collisions) deviates from the elastic limit of an ideal gas when dissipation increases?

(2) What is the phase diagram of dynamical states of such an out-of-equilibrium dissipative gas?

(3) What is the segregation mechanism in granular media composed of two types of particle species in low gravity?

(4) What is the behavior of a granular medium near the jamming transition (transition from fluid to solid) without confinement pressure of grains due to their own weight?

(5) How does sound propagate in dense granular media under low confining pressure (i.e., lower than the one provided by gravity near unjamming)?

(6) Does a dense granular medium display large-scale convection-like dynamics, like on Earth, when subjected to a thermal gradient-like forcing (i.e., two vibrating walls with different velocities)?

Some of these questions have been addressed in numerical studies but never performed in experiments. Valuable experimental data will therefore be collected, for the first time ever, with the VIP-Gran instrument.

Since these open questions concern different experimental topics, numbers of particles, protocols, and time scales, different interchangeable cells are used to address them. As a proof of concept of the instrument, we will describe below some selected results obtained with this facility during ESA parabolic flight campaigns: (A) granular gas dynamics, (B) clustering, and (C) segregation, convection, and transport.

\section{A. Granular gas dynamics}

A granular gas displays striking properties compared to molecular gas: cluster formation at high enough density, ${ }^{8,9}$ anomalous scalings of pressure ${ }^{8}$ and collision frequency, ${ }^{20}$ and non-Gaussian distribution of particle velocity. ${ }^{10,21}$ These differences are mainly ascribed to the dissipation occurring during inelastic collisions between particles. Continuous injection of energy is thus necessary to sustain a stationary state in this dissipative out-of-equilibrium system. This is usually performed experimentally by vibrating a container wall or the whole container. For such a boundary-forced system, the particle velocity distribution is not Gaussian as for an ideal gas, but a stretched exponential, and is density dependent. ${ }^{21}$ However, a spatially homogeneous forcing of a dilute granular gas leads to several major experimental differences (no cluster formation, exponential tail of the velocity distribution independent of the density). ${ }^{22}$

Here, we study with the VIP-Gran facility the analog of the dynamics of Brownian particles moving in thermalized fluids. To wit, we placed three large intruders in a driven granular gas of smaller beads that act as a surrounding fluid (see Fig. 6). Experiments were carried out in the quasi 2D cell in order to allow an easy tracking of the intruders by using the cameras. Thanks to image processing, we followed the
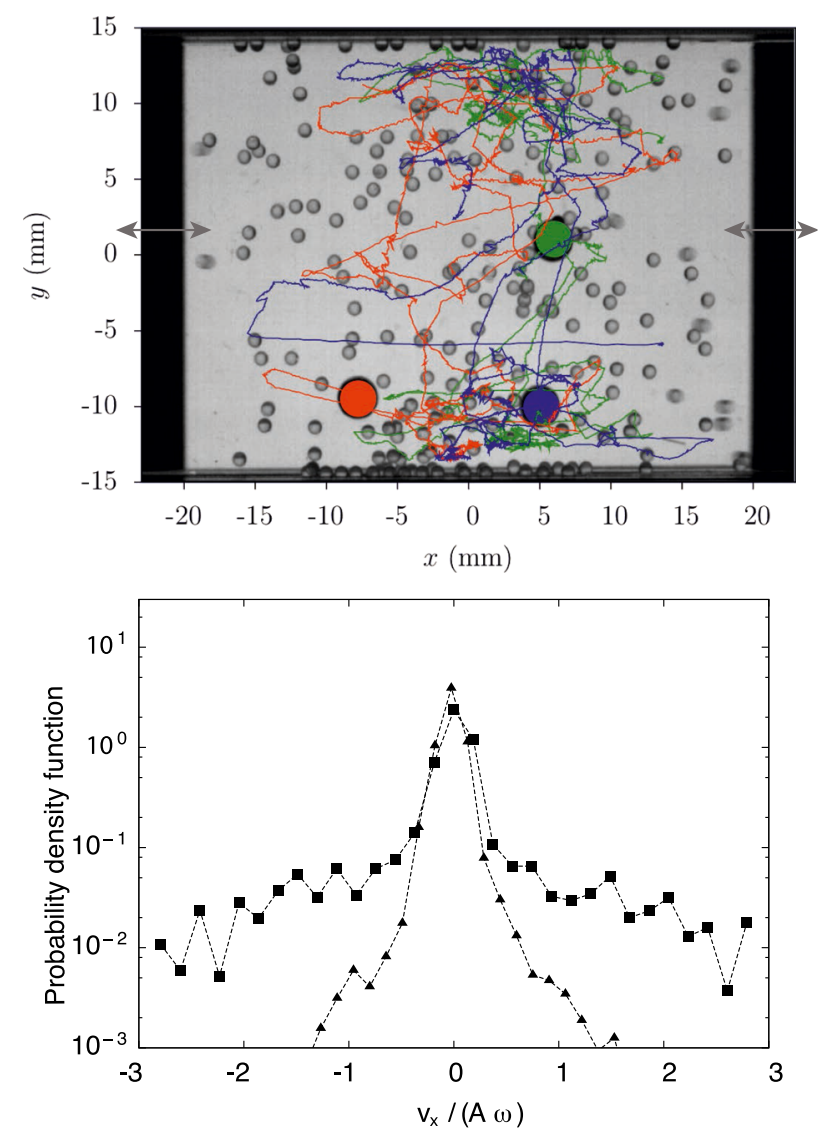

FIG. 6. Top: View of three large aluminum particles $(5 \mathrm{~mm}$ in diameter, $176.6 \mathrm{mg}$ in mass) immersed in a surrounding gas of thermalized small glass beads ( $1 \mathrm{~mm}$ in diameter, $4.08 \mathrm{mg}$ in mass) in the quasi $2 \mathrm{D}$ cell. Both pistons (left and right black parts) vibrate sinusoidally in phase opposition. Each large particle is highlighted in a color (either orange, blue, or green), as well as its respective trajectories. Bottom: PDF of the normalized velocities (along the $x$ axis) of the intruders for ( $\boldsymbol{\square})$ low and ( $\boldsymbol{\Delta})$ high numbers of small particles $\left(N_{s}=50\right.$ and $N_{s}=300$, respectively). Snapshot is taken from parabolic flight campaign ESA PFC66. Parameter range of the experiment: $f=15 \mathrm{~Hz}, A=5 \mathrm{~mm}$, packing fraction of small particles $\phi \in[1 \%, 2.5 \%]$, and $L=45 \mathrm{~mm}$. 
motion of the large particles for different numbers of small ones and different driving parameters. Figure 6 displays the trajectories of the three intruders obtained with our tracking. We then infer the mean square displacements of the intruders and the Probability Density Function (PDF) of their velocities $v_{x}$ along the $x$ axis normalized by the piston velocity $A \omega$ (with $\omega=2 \pi f$ ). We observe a non-Gaussian distribution with heavy tails for high velocities. This tail vanishes as the number of small particles increases due to the dynamical confinement of large particles by small ones. In another set of experiments, we experimentally study how a granular gas deviates from the quasi-elastic limit (ideal gas) when dissipation increases in the system. One way to do that is to increase the volume of the container at a constant particle density all things being equal, as it was shown numerically. ${ }^{23}$ By varying some parameters of the experiment (particle number and volume of the cell), one observes how the granular system deviates or tends to the quasi-elastic limit (not shown here).

\section{B. Granular clustering}

The transition from a gaseous regime to a cluster of particles for a high enough density has been evidenced in a low-gravity experiment almost 20 years ago. ${ }^{9}$ Numerical simulations have then reproduced this observation with the same parameters as in the experiment. ${ }^{24}$ However, the parameters triggering this transition are far to be fully understood. By comparing the propagation time between two collisions and the relaxation time due to dissipative collisions, a 2D theoretical model based on an energetic approach predicts the marginal curve of the phase diagram separating gas- and solidlike regimes. ${ }^{25}$ Here, with the VIP-Gran facility, we study the clustering transition in 3D to obtain the full experimental phase diagram and to compare it with the predictions. Control parameters are the number of beads $N$, the mean distance $L$ between vibrating pistons, the amplitude $A$, and frequency $f$ of the forcing. The packing fraction is then defined as $\phi=N \mathcal{V}_{g} / V \sim$ $N / L$, where $\mathcal{V}_{g}$ is the volume of a single grain and $V$ the cell volume. Different parabolic flight campaigns have been performed to cover the whole range of parameters. By means of the two perpendicular fields of view, and of image processing, the local density of particles and their velocities have been inferred in the 3D cell. As an example, Fig. 7 shows snapshots taken during PFC63 and PFC64. As one can see, starting from a gaseous regime, a cluster can be triggered by either increasing the packing fraction $\phi$ for a fixed volume (left column) or by increasing the volume for a fixed value of $\phi$ (right column). A regime of gas is discriminated from a cluster regime when the density distribution on both fields of view is found to be uniform (gas) or nonuniform (cluster). Moreover, from all the PFC data, we have obtained the full experimental phase diagram (not shown here). It is found to be well described by the model, ${ }^{25}$ except for strongly diluted and dense cases where additional phenomena are observed. The corresponding data will be deeply described elsewhere.

Another set of experiments performed within our facility concerns aspherical particle dynamics. In a dilute regime, its dynamics has been already studied in low-gravity showing non-Gaussian velocity distributions and non-equipartition. ${ }^{13}$ Here, we use granular materials composed of rods presenting an aspect ratio of about 5. By increasing the packing fraction, we observe for the first time in low-gravity the transition between a gas-like and a cluster regime of rods, as displayed in Fig. 8. The corresponding data will be deeply described elsewhere.

\section{Granular segregation, convection, and transport}

Here we give a few last examples of scientific applications that have been investigated with the VIP-Gran facility: Granular segregation, convection, and transport of particles in a partitioned cell under low gravity.

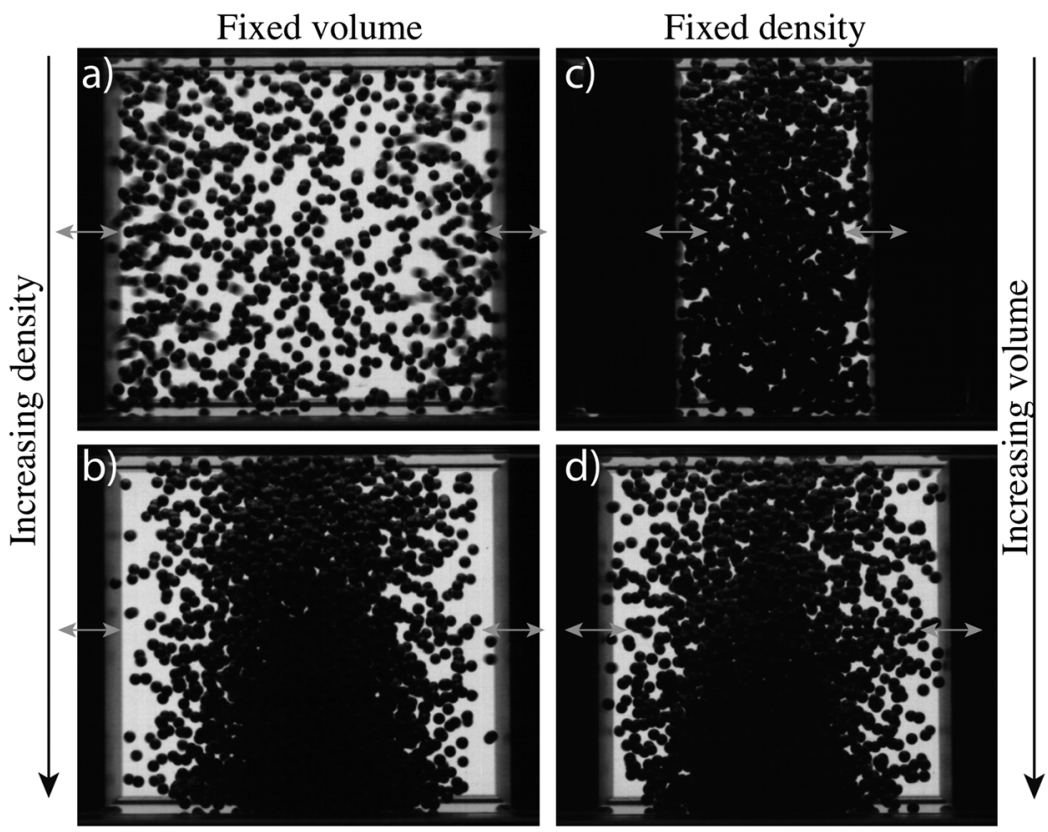

FIG. 7. Transition from a gas-like to a cluster regime of particles in low gravity in the 3D cell. Starting from a gaseous regime [(a) and (c)], a cluster can be obtained [(b) and (d)] by either (left row-PFC63) increasing the density $\phi=[1 \%$ (a) and $4 \%$ (b)] for a fixed volume ( $L=40 \mathrm{~mm}$ ) or by (right row-PFC64) increasing $L=[12.5 \mathrm{~mm}$ (c) and $27.5 \mathrm{~mm}(\mathrm{~d})]$ for a fixed value of $\phi=6.5 \%$. Both pistons (black parts) vibrate sinusoidally in phase opposition. Snapshots were taken during parabolic flight campaigns ESA PFC63 and PFC64 using the following forcing parameters: $f=20 \mathrm{~Hz}$ and $A=2 \mathrm{~mm}$. 

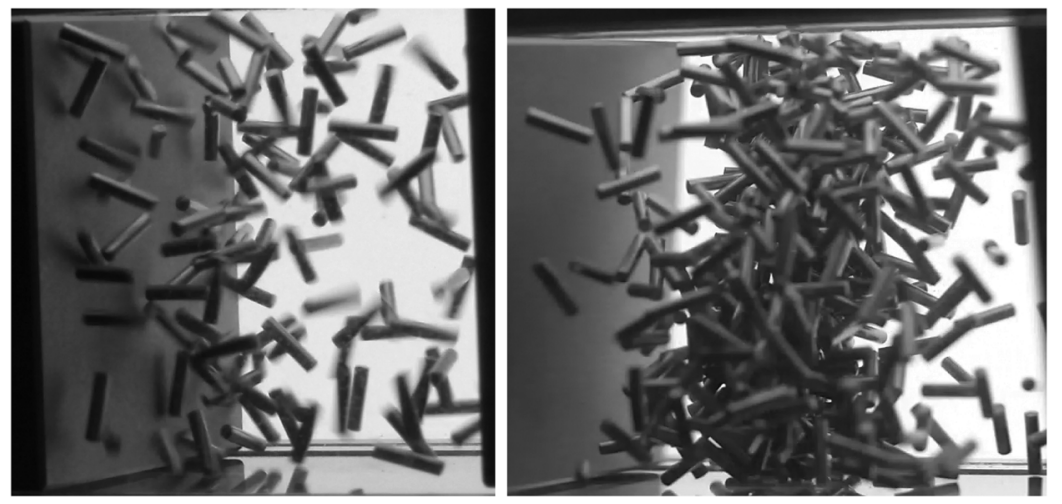

FIG. 8. Transition from a gas-like to a cluster regime of rods in low-gravity, when the packing fraction increases from $1 \%$ (left) to $3 \%$ (right). Experiments were performed in the 3D cell during the parabolic flight campaign ESA PFC66. Driving parameters and length in these snapshots are $f=15 \mathrm{~Hz}, A=5 \mathrm{~mm}$, and $L=40 \mathrm{~mm}$. Stainless steel rods (issued from bearings) are $1 \mathrm{~mm}$ in diameter and $4.3 \mathrm{~mm}$ in length.

\section{Segregation}

On Earth, segregation of a granular medium composed of different particle species is well-known (and often called the Brazil nut effect) and is due to different mechanisms related to gravity, either convection by friction of particles on boundaries ${ }^{26}$ or percolation of small particles beneath large ones. ${ }^{27}$ In reduced gravity conditions, the segregation mechanism is unknown and the relevant control parameters are not yet established. ${ }^{3}$ The origin of large-scale particle segregation observed on the asteroid regolith surfaces is a matter of intense debate..$^{28,29}$ Recently, collisional segregation has been proposed in this context ${ }^{29}$ since a loose granular bed plays the role of impact absorbers either with gravity ${ }^{30}$ or in the absence of gravity. ${ }^{31}$

Here, with the VIP-Gran facility, a mixture of small and large grains is subjected to vibrations in low gravity. Depending of the number $N_{s}$ of small particles and the number $N_{l}$ of large ones, different dynamics have been observed in the quasi 2D cell. In a dilute case, both particle types behave like gases homogeneously distributed [Fig. 9(a)]. When additional small beads are injected into the cell, large beads are then forced to the cluster in a strip in the center of the cell, thus leading to segregation [Fig. 9(b)]. This effect is due to the dissipative nature of collisions. For high enough fillings (of both particle types), we observed for the first time in low gravity a pattern in the center of the cell, constituted of alternative strips of small and large particles, ${ }^{32}$ as it was predicted numerically ${ }^{33,34}$ and theoretically. ${ }^{35}$

\section{Convection}

On Earth, convection in granular media is related to a spatial gradient of kinetic energy that, combined with gravity, produces a buoyancy-like force. When dissipation is large enough, this force can trigger the convection. ${ }^{7,36}$ This mechanism should not be confused with air effect triggering convection rolls for submillimeter particles ${ }^{5,37}$ or with convection induced by particle friction on boundaries for submillimetric particles. ${ }^{3,26,38}$ In low-gravity conditions, with no boundary or air effect, convection has only been observed numerically. ${ }^{33}$ With the VIP-Gran facility, one can apply different phases and velocities on each of the pistons to mimic a thermal-like gradient forcing. The corresponding cold and hot walls are expected to drive the system into a convectivelike motion due to the generated spatial gradients of the kinetic energy. ${ }^{39}$ Such large-scale convective motions have been observed in the 3D cell, during PFC experiments, within a mixture of large and small beads. However, the impact of the $g$-jitters on the phenomenon must still be clarified. This coarsening dynamics and the appearance of vortex-like structures will be further analyzed. In future, much longer experiments with a better level of low gravity will thus be performed in space (see Sec. III D).

\section{Handling a granular medium in low gravity}

On Earth, pouring a granular material is an easy way to handle it. In the absence of gravity, applying vibrations
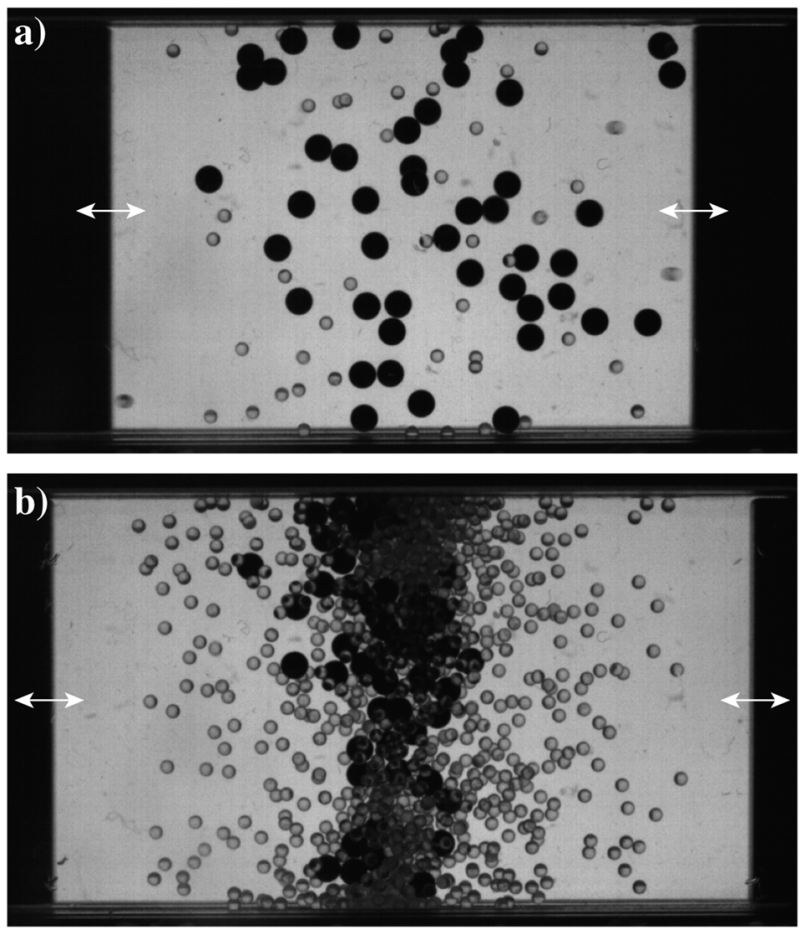

FIG. 9. Segregation of a mixture of large bronze and small glass beads in the $3 \mathrm{D}$ cell. In a dilute case $\left(N_{L}=50, N_{s}=50\right)$, both particle types behave like a gas homogeneously distributed in the cell. (a) With additional small particles $\left(N_{L}=50, N_{s}=1000\right)$, the latter force the large particles to accumulate in a stripe at the center of the cell, thus inducing segregation. (b) Both pistons (black parts) vibrates sinusoidally in phase opposition. Snapshots were taken during parabolic flight campaign ESA PFC65 with forcing parameters $f=15 \mathrm{~Hz}$ and $A=5 \mathrm{~mm}$. 
to the medium within partitioned cells with slits is a technique to manipulate it. However, the mechanisms leading to the exchange of particles between the compartments are poorly understood in the absence of gravity. ${ }^{40-43}$ Within a box made of two connected compartments, the separation of a granular system into a cold and dense region on the one hand and a hot and dilute part on the other hand is usually called the Maxwell Demon experiment. On Earth, the apparent intrusion of a Maxwell Demon has been investigated theoretically, ${ }^{44}$ experimentally, ${ }^{45}$ and numerically. ${ }^{46}$ The particle fluxes between compartments are related to the ratio between the gravitational potential energy of particles and the granular temperature both taken at the altitude of the slit. ${ }^{40}$ In a low gravity, this control parameter of the phase separation does not hold up. Thus, investigations in low-gravity experiments involving compartmented systems are foreseen for next PFCs within the VIP-Gran facility. This study will be complementary to the Chinese SJ-10 satellite mission ${ }^{47}$ and will be guided by previous numerical simulations with mono-disperse ${ }^{42}$ or bidisperse $^{43}$ particles in compartmented systems in the absence of gravity.

\section{Future experiments in the International Space Station}

The VIP-Gran facility was selected in early 2017 for flying on the International Space Station (ISS) in 2019. The ISS version of the instrument is currently in development. Excellent low-gravity conditions $\left(\sim 10^{-5} \mathrm{~g}\right)$ are indeed needed to avoid $g$-jitters, as observed in previous PFC notably for cooling and convection experiments. Long time duration experiments ( $\gg 22$ s of PFC) are also needed to perform numerous iterative experiments to reach statistically convergent observations or for phenomena with long transients. Since the beginning of the project, the VIP-Gran instrument has been conceived for an ISS usage and, accordingly, its design was orientated toward versatility, full automatization, and modularity through interchangeable cells. Several experiments not discussed here are planned with the ISS version of the instrument, such as dynamics of granular cooling (when the forcing is stopped), ${ }^{48}$ sound propagation, and rheology near the onset of jamming in dense granular media.

\section{CONCLUSION}

To summarize, we have presented an experimental setup dedicated to the study of granular media in a low-gravity environment in three or two dimensions. This instrument is capable of conducting experiments over a large range of granular packing fractions from diluted to dense regimes (up to approximately 60\%). The forcing mechanism is composed by two opposite walls of the cell driven by selected stimuli (sine, single-pulse, quasi-static ramp, etc.). The setup is instrumented by means of high-speed cameras in two perpendicular fields of view and accelerometers and impact sensors that allow us to infer quantitative measurements. The cell volume, the number of particles, and the amplitude, frequency, and phase of the driving are control parameters. Our preliminary results with modular geometries, based on different interchangeable cells, demonstrate the versatility of our setup. Our system thus provides a unique facility to perform experiments on granular media in reduced gravity conditions. Several open questions are currently investigated with this facility related to granular gases, segregation, convection, and transport of grains in a low gravity. Original scientific contributions as well as new applications for space exploration are thus expected.

An updated version of this facility is currently in development for the International Space Station to obtain excellent low-gravity conditions and a long duration of experimentations to study, notably, the cooling of a granular gas, convection, sound propagation in dense granular media under a ultra-low applied pressure, and rheology near the onset of the jamming transition without the confinement pressure of grains due to their weight.

\section{ACKNOWLEDGMENTS}

We thank M. Braibanti, O. Minster, and V. Köhne from ESA for fruitful discussions and for the flight opportunity. VIP-Gran-PF was built by DTM Technologies ${ }^{\mathrm{TM}}$ (Modena, Italy). We thank A. Pellegrini, L. Recanatesi, and D. Santachiara for their experimental support on PFC64. We thank the support of Novespace during parabolic flight campaigns (PFC64 to PFC67). E.O. thanks Prodex (Belspo) for financial support. Y.G., C.L., and F.P. thank P. Evesque for collaboration and thank CNES for both financial support and access to their PFC. This work was funded by ESA Topical Team No. 4000103461, CCN1, and CCN2.

${ }^{1}$ B. Andreotti, Y. Forterre, and O. Pouliquen, Granular Media: Between Fluid and Solid (Cambridge University Press, Cambridge, 2013); J. Duran, Sands, Powders, and Grains (Springer-Verlag, New York, 2000); P. Coussot, Rheometry of Pastes, Suspensions, and Granular Materials: Applications in Industry and Environment (John Wiley and Sons, 2005).

${ }^{2}$ F. Spahn, J. Schmidt, and M. Sremcevic, Lect. Notes Phys. 557, 507 (2000). ${ }^{3}$ C. Güttler, I. von Borstel, R. Schräpler, and J. Blum, Phys. Rev. E 87, 044201 (2013).

${ }^{4}$ See http://www.planetaryresources.com for Planetary Resources, Inc. (formerly Arkyd Astronautics) is an american start-up company aiming a profit-making mining of near earth asteroids.

${ }^{5}$ C. Laroche, S. Douady, and S. Fauve, J. Phys. 50, 699 (1989).

${ }^{6}$ S. Fauve, S. Douady, and C. Laroche, J. Phys. Colloq. 50, C3-187 (1989); F. Melo, P. Umbanhowar, and H. L. Swinney, Phys. Rev. Lett. 72, 172 (1994).

${ }^{7}$ S. McNamara and S. Luding, Phys. Rev. E 58, 813-822 (1998)

${ }^{8}$ E. Falcon, S. Fauve, and C. Laroche, Eur. Phys. J. B 9, 183 (1999).

${ }^{9}$ E. Falcon, R. Wunenburger, P. Evesque, S. Fauve, C. Chabot, Y. Garrabos, and D. Beysens, Phys. Rev. Lett. 83(2), 440 (1999).

${ }^{10}$ S. Luding, R. Cafiero, and H. J. Herrmann, "Driven granular gases," in Granular Gas Dynamics, Lecture Notes in Physics, Vol. 624, edited by T. Pöschel and N. Brillantov (Springer Verlag, Berlin, 2003).

${ }^{11}$ S. McNamara, Phys. Fluids A 5, 3056 (1993); I. Goldhirsch and G. Zanetti, Phys. Rev. Lett. 70, 1619 (1993); N. Brilliantov, C. Salueña, T. Schwager, and T. Pöschel, ibid. 93, 134301 (2004); S. Luding and H. J. Herrmann, Chaos 9(3), 673-681 (1999); S. Miller and S. Luding, Phys. Rev. E 69(3), 031305 (2004); S. Gonzalez, A. R. Thornton, and S. Luding, Eur. Phys. J.: Spec. Top. 223(11), 2205-2225 (2014).

${ }^{12}$ S. McNamara and W. R. Young, Phys. Fluids A 4, 496 (1992); S. Luding and S. McNamara, Granular Matter 1(3), 113-128 (1998).

${ }^{13}$ K. Harth, U. Kornek, T. Trittel, U. Strachauer, S. Höme, K. Will, and R. Stannarius, Phys. Rev. Lett. 110, 144102 (2013); K. Harth, T. Trittel, K. May, S. Wegner, and R. Stannarius, Adv. Space Res. 55, 1901 (2015).

${ }^{14}$ K. Harth, T. Trittel, S. Wegner, and R. Stannarius, Phys. Rev. Lett. 120 , 214301 (2018). 
${ }^{15}$ G. Bossis, Y. Grasselli, and O. Volkova, J. Phys.: Condens. Matter 16(18), 3279 (2004).

${ }^{16}$ C. H. Liu and S. R. Nagel, "Sound in sand," Phys. Rev. Lett. 68, 2301 (1992); X. Jia, C. Caroli, and B. Velicky, ibid. 82, 1863 (1999); B. Gilles and C. Coste, ibid. 90, 174302 (2003); S. Wildenberg, Y. Yang, and X. Jia, Granular Matter 17, 419 (2015); S. Wildenberg, A. Tourin, and X. Jia, Europhys. Lett. 115, 34005 (2016); R. K. Shrivastava and S. Luding, Nonlin. Processes Geophys. 24, 435-454 (2017); A. Merkel and S. Luding, Int. J. Solids Struct. 106-107, 91-105 (2017); O. Mouraille and S. Luding, Ultrasonics 48, 498-505 (2008).

${ }^{17}$ T. S. Majmudar and R. P. Behringer, Nature 435, 1079 (2005); T. S. Majmudar, M. Sperl, S. Luding, and R. P. Behringer, Phys. Rev. Lett. 98, 058001 (2007); R. Harich, T. Darnige, E. Kolb, and E. Clément, Europhys. Lett. 96, 54003 (2011); D. Bi, J. Zhang, B. Chakraborty, and R. P. Behringer, Nature 480, 355 (2011); J. Ren, J. A. Dijksman, and R. P. Behringer, Phys. Rev. Lett. 110, 018302 (2013); H. Mizuno, L. E. Silbert, and M. Sperl, ibid. 116, 068302 (2016); S. Luding, Nat. Phys. 12, 531-532 (2016); N. Kumar and S. Luding, Granular Matter 18, 58 (2016).

${ }^{18}$ See http://www.spacegrains.org for latest news on VIP-Gran.

${ }^{19}$ Airbus A310 Zero-G is managed and operated by the French company Novespace from the Bordeaux-Mérignac airport (France).

${ }^{20}$ E. Falcon, S. Aumaître, P. Evesque, F. Palencia, C. Lecoutre-Chabot, S. Fauve, D. Beysens, and Y. Garrabos, Europhys. Lett. 74, 830 (2006).

${ }^{21}$ F. Rouyer and N. Menon, Phys. Rev. Lett. 85, 3676 (2000); W. Losert, D. G. W. Cooper, J. Delour, A. Kudrolli, and J. P. Gollub, Chaos 9, 682 (1999); J. S. Olafsen and J. S. Urbach, Phys. Rev. E 60, R2468(R) (1999); A. Kudrolli and J. Henry, ibid. 62, R1489 (2000); J. S. van Zon, J. Kreft, D. I. Goldman, D. Miracle, J. B. Swift, and H. L. Swinney, ibid. 70, 040301(R) (2004); H.-Q. Wang, K. Feitosa, and N. Menon, ibid. 80, 060304(R) (2009); S. Tatsumi, Y. Murayama, H. Hayakawa, and M. Sano, J. Fluid Mech. 641, 521 (2009); S. E. Episov and T. Pöschel, J. Stat. Phys. 86, 1385 (1997); C. Scholz and T. Pöschel, Phys. Rev. Lett. 118, 198003 (2017).

${ }^{22}$ E. Falcon, J.-C. Bacri, and C. Laroche, Europhys. Lett. 103, 64004 (2013); Phys. Rev. Fluids 2, 102601(R) (2017)

${ }^{23}$ S. Aumaître, J. Farago, S. Fauve, and S. McNamara, Eur. Phys. J. B 42, 255 (2004).

${ }^{24}$ E. Opsomer, F. Ludewig, and N. Vandewalle, Phys. Rev. E. 84, 051306 (2011); J. Phys.: Conf. Ser. 327, 012035 (2011).

${ }^{25}$ E. Opsomer, F. Ludewig, and N. Vandewalle, Europhys. Lett. 99, 40001 (2012).

26 J. B. Knight, H. M. Jaeger, and S. R. Nagel, Phys. Rev. Lett. 70, 3728 (1993); R. Khosropour, J. Zirinsky, H. K. Pak, and R. P. Behringer, Phys. Rev. E 56, 4467 (1997).

${ }^{27}$ J. C. Williams, Powder Technol. 52, 245 (1976); A. Rosato, K. J. Strandburg, F. Prinz, and R. H. Swendsen, Phys. Rev. Lett. 58, 1038 (1987); H. M. Jaeger, S. R. Nagel, and R. P. Behringer, Rev. Mod. Phys. 68, 1259 (1996).

${ }^{28}$ N. Murdoch, P. Sánchez, S. R. Schwartz, and H. Miyamoto, in Asteroids IV, edited by M. Patrick, F. W. DeMeo, and W. F. Bottke (University of Arizona Press, Tucson, AZ, 2015), p. 767.
${ }^{29}$ T. Shinbrot, T. Sabuwala, T. Siu, M. Vivar Lazo, and P. Chakraborty, Phys. Rev. Lett. 118, 111101 (2017).

${ }^{30}$ F. Pacheco Vázquez and S. Dorbolo, Sci. Rep. 3, 2158 (2013).

${ }^{31}$ S. McNamara and E. Falcon, Powder Technol. 182, 232 (2008).

${ }^{32}$ E. Opsomer, M. Noirhomme, N. Vandewalle, E. Falcon, and S. Merminod, npj Microgravity 3, 1 (2017).

${ }^{33}$ S. Luding, O. Strauss, and S. McNamara, "Segregation of polydisperse granular media in the presence of a temperature gradient," in IUTAM Symposion on Segregation in Granular Flows 297-303, edited by A. D. Rosato and D. L. Blackmore (Kluwer Academic Publishers, 2000).

${ }^{34}$ E. Opsomer, N. Vandewalle, M. Noirhomme, and F. Ludewig, Eur. Phys. J. E 37, 115 (2014).

${ }^{35}$ D. Serero, S. H. Noskowicz, M. L. Tan, and I. Goldhirsch, Eur. Phys. J.: Spec. Top. 179, 221 (2009).

${ }^{36}$ R. Ramírez, D. Risso, and P. Cordero, Phys. Rev. Lett. 85, 1230 (2000); R. D. Wildman, J. M. Huntley, and D. J. Parker, ibid. 86, 3304 (2001); E. Khain and B. Meerson, Phys. Rev. E 67, 021306 (2003); D. Paolotti, A. Barrat, U. Marini Bettolo Marconi, and A. Puglisi, ibid. 69, 061304 (2004).

${ }^{37}$ H. K. Pak and R. P. Behringer, Phys. Rev. Lett. 71, 1832 (1993); K. Kumar, E. Falcon, K. M. S. Bajaj, and S. Fauve, Physica A 270, 97 (1999); A. Garcimartín, J. M. Pastor, R. Arévalo, and D. Maza, Eur. Phys. J.: Spec. Top. 146, 331 (2007).

${ }^{38}$ N. Murdoch, B. Rozitis, K. Nordstrom, S. F. Green, P. Michel, T.-L. de Lophem, and W. Losert, Phys. Rev. Lett. 110, 018307 (2013).

${ }^{39}$ P. Eshuis, K. van der Weele, M. Alam, H. J. van Gerner, M. van der Hoef, H. Kuipers, S. Luding, D. van der Meer, and D. Lohse, Granular Matter 15(6), 893-911 (2013).

${ }^{40}$ N. Isert, C. C. Maaß, and C. M. Aegerter, Eur. Phys. J. E 28, 205 (2009).

${ }^{41}$ J. J. Brey, F. Moreno, R. García-Rojo, and M. J. Ruiz-Montero, Phys. Rev. E 65, 011305 (2001).

${ }^{42}$ Y. Li, M. Hou, and P. Evesque, J. Phys.: Conf. Ser. 327, 012034 (2011); E. Opsomer, M. Noirhomme, N. Vandewalle, and F. Ludewig, Phys. Rev. E 88, 012202 (2013); M. Noirhomme, E. Opsomer, N. Vandewalle, and F. Ludewig, Eur. Phys. J. E 38, 9 (2015).

${ }^{43}$ W. Wang, Z. Zhou, J. Zong, and M.-Y. Hou, Chin. Phys. B 26, 044501 (2017).

${ }^{44}$ J. Eggers, Phys. Rev. Lett. 83, 5322 (1999).

${ }^{45}$ K. van der Weele, D. van der Meer, M. Versluis, and D. Lohse, Europhys. Lett. 53, 328 (2001).

${ }^{46}$ A. Barrat and E. Trizac, Mol. Phys. 101, 1713 (2003); M. Hou, H. Tu, R. Liu, Y. Li, K. Yu, P.-Y. Lai, and C. K. Chan, Phys. Rev. Lett. 100, 068001 (2008); S. Shah, Y. C. Li, F. F. Cui, Q. Zhang, P. Evesque, and M. Hou, Chin. Phys. Lett. 29, 034501 (2012).

${ }^{47}$ M. Hou, private communication (2018).

${ }^{48}$ C. C. Maaß, N. Isert, G. Maret, and C. M. Aegerter, Phys. Rev. Lett. 100, 248001 (2008); Y. Grasselli, G. Bossis, and G. Goutallier, Europhys. Lett. 86, 60007 (2009); S. Tatsumi, Y. Murayama, H. Hayakawa, and M. Sano, J. Fluid Mech. 641, 521 (2009); D. Heißelmann, J. Blum, H. J. Fraser, and K. Wolling, Icarus 206, 424 (2010); K. Harth, T. Trittel, S. Wegner, and R. Stannarius, EPJ Web Conf. 140, 04008 (2017). 